\title{
Design of Photonic Crystal Fiber Capable of Carrying Multiple Orbital Angular Momentum Modes Transmission
}

\author{
Xizheng Ke ${ }^{1,2}$, Shasha Wang ${ }^{*}$ \\ ${ }^{1}$ Faculty of Automation and Information Engineering, Xi'an University of Technology, Xi'an, China \\ ${ }^{2}$ Shaanxi Civil-Military Integration Key Laboratory of Intelligence Collaborative Networks, Xi'an, China \\ Email: ^451162578@qq.com
}

How to cite this paper: Ke, X.Z. and Wang, S.S. (2020) Design of Photonic Crystal Fiber Capable of Carrying Multiple Orbital Angular Momentum Modes Transmission. Optics and Photonics Journal, 10, 49-63.

https://doi.org/10.4236/opj.2020.104005

Received: March 4, 2020

Accepted: April 7, 2020

Published: April 10, 2020

Copyright $\odot 2020$ by author(s) and Scientific Research Publishing Inc. This work is licensed under the Creative Commons Attribution International License (CC BY 4.0).

http://creativecommons.org/licenses/by/4.0/

\begin{abstract}
For the traditional photonic crystal fibers with circular air holes, rectangular air holes are added to the fiber cladding. The periodic arrangement of the inner rectangular air holes allows the fiber structure to better match the annular mode field distribution of the vortex beam. The fiber structure was analyzed and calculated by COMSOL Multiphysics 5.4 finite element software, and the characteristics of fiber were analyzed, such as the dispersion, confinement loss, effective mode area and nonlinear coefficient. The results reveal that the photonic crystal fiber structure capable of carrying 50 orbital angular momentum (OAM) modes at the wavelength of 1.15 to $2.0 \mu \mathrm{m}(850 \mathrm{~nm})$. The effective refractive index difference $\Delta n_{\text {eff }}$ between vector modes can reach $1 \times$ $10^{-3}$, and larger difference can effectively separate the vector modes and improve the transmission performance of OAM modes. Moreover, the fiber has good performance, such as flat dispersion distribution of the low-order modes, low confinement loss below $10^{-9} \mathrm{~dB} \cdot \mathrm{m}^{-1}$, large effective mode field area and small nonlinear coefficient in the $850 \mathrm{~nm}$ wavelength range. Therefore, this fiber structure can be applied to the high-capacity communication system of fiber multiplexing OAM. In addition, the good characteristics of this fiber structure are of great significance for the transmission of vortex beam in fiber.
\end{abstract}

\section{Keywords}

Fiber Optics Communication, Photonic Crystal Fiber, Orbital Angular Momentum Modes, Mode Effective Refraction Index

\section{Introduction}

With the rapid changes in the informatization of modern society, the communica- 
tion technology has developed toward high speed and large capacity [1]. The vortex beam is a new type of beam with helical phase wavefront, and OAM modes with different topological charges are orthogonal in space, which can be used as independent channels. So the vortex beam can realize the multi-channel transmission of the information, which fundamentally improves the communication capacity of the free-space optical communication system [2] [3]. Therefore, the generation of high-quality vortex beams is a key issue in the research of orbital angular momentum (OAM) multiplexed communication system. In addition, the vortex beams generated by the fiber in many generation methods can simplify the optical structure and have higher phase purity. And the combination of the fiber and the vortex beam can not only improve the performance of the fiber, but also expand the application of the fiber. Generally, the difference in effective refractive index between vector modes in fiber is too small, the modes will occur crosstalk and merging each other during propagation to form a linearly polarization (LP) mode, and the OAM mode cannot be transmitted stably in such waveguides [4]. In summary, it is particularly pivotal to design a fiber that can support multiple OAM modes, and ensure the stable transmission of vortex beams in fiber.

In recent years, various fiber structures supporting OAM modes transmission have been studied. Fibers such as air-core step fiber [5] [6], graded-index fiber [7] and multi-core fiber [8] don't have enough adjustable parameters to ensure the purity of the OAM mode. Moreover, the effective refractive index difference between the vector modes is mostly on the order of $10^{-4}$, so that the number of OAM modes supported by the fiber is small. Compared with the traditional fiber structure, the photonic crystal fiber (PCF) is more conducive to the design of various new functional fibers because of its many adjustable parameters, such as the low loss, excellent nonlinearity and dispersion, etc. [9]. In 2012, Yue et al. [10] designed a regular hexagonal PCF with the cladding material of $\mathrm{As}_{2} \mathrm{~S}_{3}$, which can only support 2 OAM modes transmission, but this fiber has larger dispersion and confinement loss, which is not conducive to stable transmission of OAM modes. Wong et al. [11] twisted the PCF to generate a variety of OAM modes, which had the advantage of generating abundant OAM modes. In 2016, Tian et al. [12] designed a circular ring PCF structure that can support 26 OAM modes transmission in the core, and studied the characteristics of dispersion and confinement loss. In 2018, Nandam et al. [13] proposed a spiral PCF with spirally arranged air holes that can support 14 OAM modes, and the effect of ellipticity on the index of fiber mode was discussed. But this fiber is relatively complex, which is not conducive to large-scale applications. Bai et al. [14] proposed a PCF with rectangular air holes that can support 46 OAM modes, which greatly increased the number of modes. In summary, using PCF to transmit the OAM mode, the number of the modes and inter-mode crosstalk is still difficult to affect its application.

In this study, by optimizing and modifying the size and spacing of air holes, a PCF with a combination of rectangular and circular air holes was proposed. The 
fiber structure was analyzed and calculated by COMSOL Multiphysics 5.4 finite element software. And the fiber characteristics were studied in detail. Also, the fiber has good performance, such as flat dispersion distribution, low confinement loss, large effective mode field area and small nonlinear coefficient in the $850 \mathrm{~nm}$ wavelength range.

\section{Theoretical Basis and Design Principle}

\subsection{Theoretical Basis of the Fibers}

The fiber is a circularly symmetrical dielectric optical waveguide. There are four vector modes, including TE mode, TM mode, HE mode and EH mode [15]. Among them, the HE and EH mode both have odd and even mode states, and there is a phase difference of $\pm \pi / 2$ between the two mode states [16]. Generally, the effective refractive index difference between vector modes is small, and the modes will occur crosstalk during propagation to form a LP mode [17] [18]. Similar to the LP mode, the OAM modes are also composed of vector modes in the fiber. When the effective refractive index difference between the modes is greater than $1 \times 10^{-4}$, the vector modes will be superimposed to generate the OAM mode.

The OAM modes are generally composed of the odd-mode and even-mode linear superposition of the same order HE mode or $\mathrm{EH}$ mode in the fiber, which can be expressed as [19]:

$$
\begin{aligned}
& \mathrm{OAM}_{ \pm l, m}^{ \pm}=\mathrm{HE}_{l+1, m}^{\text {even }} \pm j \mathrm{HE}_{l+1, m}^{\text {odd }} \\
& \mathrm{OAM}_{ \pm l, m}^{\mp}=\mathrm{EH}_{l-1, m}^{\text {even }} \pm j \mathrm{EH}_{l-1, m}^{\text {odd }}
\end{aligned}
$$

where $l$ represents the topological charge, and $m$ means the radial mode order. The superscript " \pm " of $\mathrm{OAM}_{ \pm l, m}^{ \pm}$means the spin state of the OAM mode and the subscript " \pm " means the direction of the wavefront rotation. The "even" and "odd" represent even mode and odd mode, respectively.

According to Equation (1) and Equation (2), it is known that $\mathrm{HE}_{l+1, m}$ or $\mathrm{EH}_{l-1, m}$ can be synthesized into the $\mathrm{OAM}_{l, m}$ mode. The $\mathrm{OAM}_{l, m}$ mode formed by the superposition of $\mathrm{HE}_{l+1, m}^{\text {even }}$ and $\mathrm{HE}_{l+1, m}^{\text {odd }}$ has the same circular polarization and field rotation direction, while the $\mathrm{OAM}_{l, m}$ mode formed by the superposition of $\mathrm{EH}_{l-1, m}^{\text {even }}$ and $\mathrm{EH}_{l-1, m}^{\text {odd }}$ has the opposite behavior.

\subsection{Photonic Crystal Fiber Structure}

For effectively separate the vector modes and generate multiple OAM modes, the basic objectives of the fiber structure design [12] mainly include: 1) as many the OAM modes as possible can be generated, and the effective refractive index differences between the vector modes should large enough $\left(>1 \times 10^{-4}\right)$. 2) The fiber structure should be matched with the annular distribution of the OAM mode field, and it has a excellent fiber performance in $\mathrm{C}+\mathrm{L}$ band.

In this study, the PCF structure is improved from two aspects of fiber cladding air holes and fiber structure. The cladding air holes are set in a circular annular 
distribution around the fiber core, which is beneficial to the generation of OAM modes of annular mode field distribution. In addition, the cladding air hole is set to a combination of rectangle and circle in the structure to provide a higher air filling rate. This method not only ensures a large refractive index difference in the fiber, but also prevents the leakage of higher-order modes. when the cladding of the PCF uses a rectangular air hole, the structure is not easily deformed, and the PCF has more stable characteristics [20] [21]. With the development of technology, rectangular air holes can be realized by methods such as sol, gel, casting and photolithography [22] [23], and the PCF with rectangular air holes can be fabricated.

Figure 1 shows the distance from the core to the cladding air hole is unchanged, and the effect of the core radius on the topological charge, radial order and the mode effective refractive index difference between the OAM modes at a wavelength of $1.55 \mu \mathrm{m}$. As shown in Figure 1(a), when the core radius $r_{0}$ gradually increases, the second-order radial mode disappears gradually. When the core radius $r_{0}$ is $8 \mu \mathrm{m}$, all the second-order radial modes disappear completely. In order to facilitate modular division multiplexing, this study only considers the first-order radial mode, taking $m=1$. When the core radius $r_{0}$ changes in the

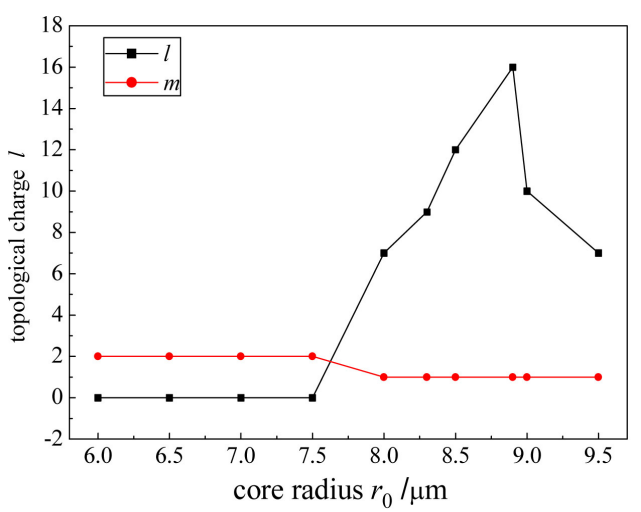

(a)

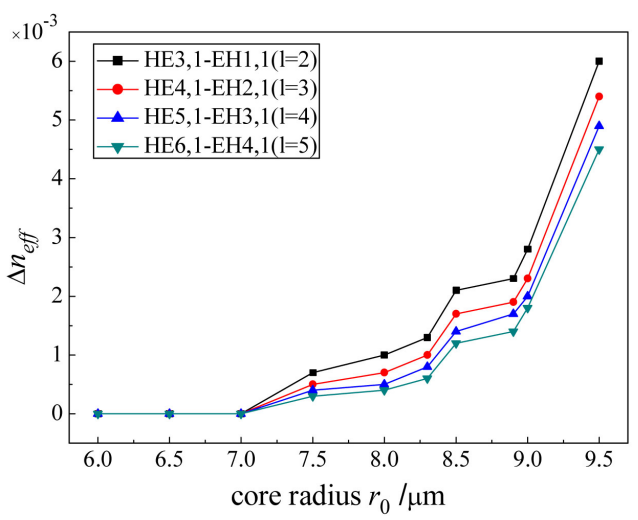

(b)

Figure 1. Variation of OAM mode with core radius $r_{0}$. (a) topological charge number $I$ and $m$ of $\mathrm{OAM}_{l, m}$ mode with core radius $r_{0}$; (b) effective refractive index difference of vector mode with core radius $r_{0}$. 
range of 6.0 to $9.5 \mu \mathrm{m}$, the number of OAM modes increases at first and then decreases gradually, and the field distribution of the vector mode becomes more and more clear, which is beneficial to distinguish the vector modes of each order. When the core radius $r_{0}$ is $8.9 \mu \mathrm{m}$, the number of OAM modes is the highest and reaches the peak value. Figure 1(b) shows the relationship between the core radius and the effective refractive index difference of the vector modes. It can be seen that the difference between the vector modes becomes larger with the increase of the core radius $r_{0}$. Combining Figure 1(a) and Figure 1(b), considering both the number of OAM modes and the effective refractive index difference between vector modes, the core radius $r_{0}$ is $8.9 \mu \mathrm{m}$. Therefore, in the optimized design of the fiber structure, the effective refractive index differences between the vector modes cannot be blindly increased, and the number of OAM modes must be considered.

The cross-section of the improved PCF structure is shown in Figure 2. The fiber core is a large air hole with the air hole radius is $r_{0}=8.9 \mu \mathrm{m}$ and the thickness of annular high refractive index region is $d=2.5 \mu \mathrm{m}$. The cladding is arranged as four layers of air hole ring layer outward around the center of the circle, and the distance between cladding layers is $\Lambda=0.3 \mu \mathrm{m}$. The length of rectangular air hole is $a=1.5 \mu \mathrm{m}$, the width is $b=1.2 \mu \mathrm{m}$, while the diameter of the circular air hole is $d_{1}=1.8 \mu \mathrm{m}$. The substrate material is silica $\left(\mathrm{SiO}_{2}\right)$, whose refractive index is $n_{1}=1.444$ at $1.55 \mu \mathrm{m}$, and air hole is $n_{2}=1$.

\section{Simulation Results}

The PCF structure designed in this study is calculated and analyzed by using the finite element software, the mode field distribution and the OAM modes supported in designed PCF were obtained.

\subsection{The Mode Field Distribution}

Figure 3 and Figure 4 show the normalized intensity distributions and the field intensity distribution in the Ez direction of the partial vector modes in the PCF at the wavelength of $1.55 \mu \mathrm{m}$, respectively. Figure 3 shows that the modes in the
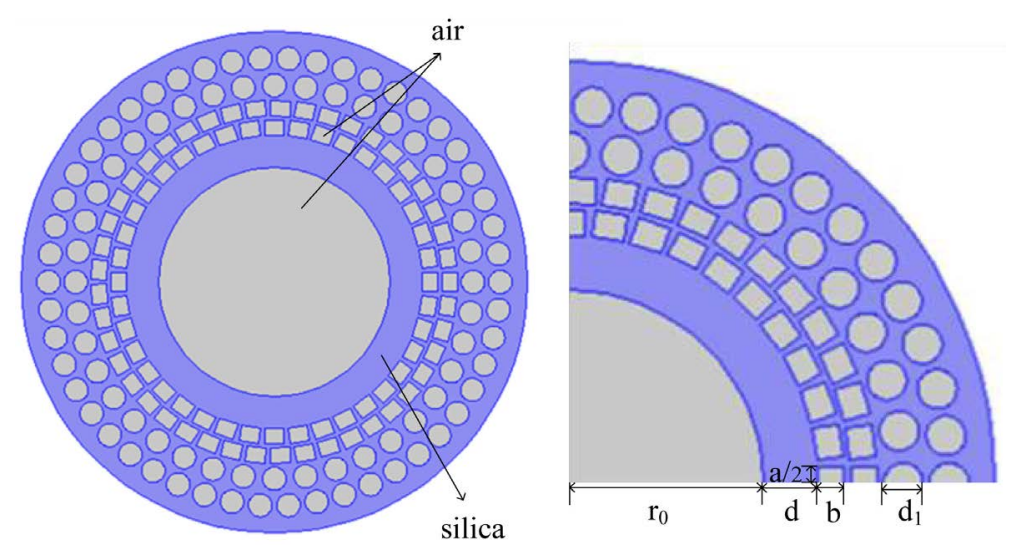

Figure 2. Schematic diagram of cross section in the PCF. 

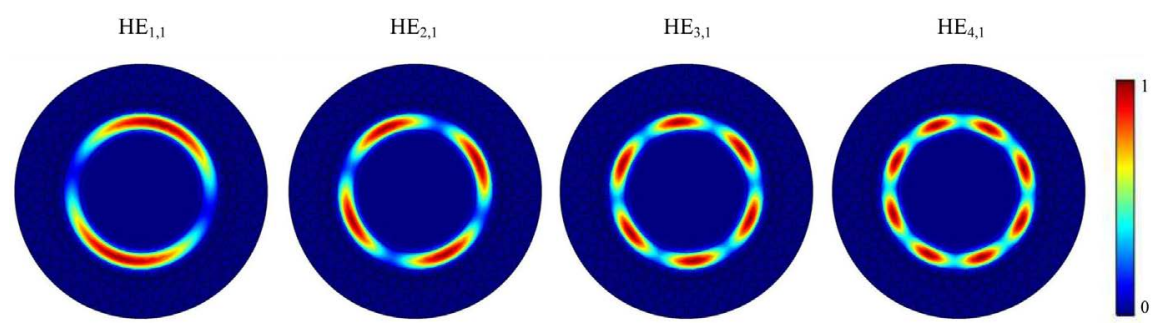

Figure 3. Normalized intensity distributions of the partial modes in the proposed PCF.

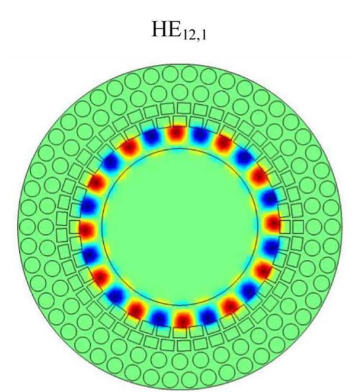

$\mathrm{EH}_{12,1}$

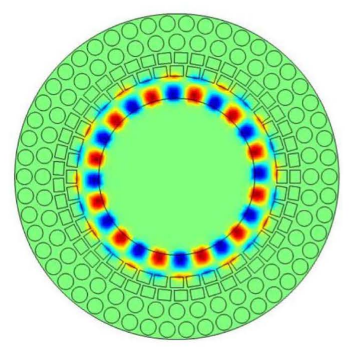

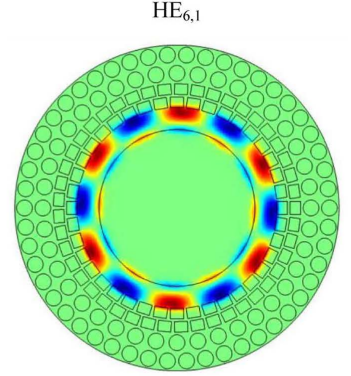

$\mathrm{EH}_{6,1}$

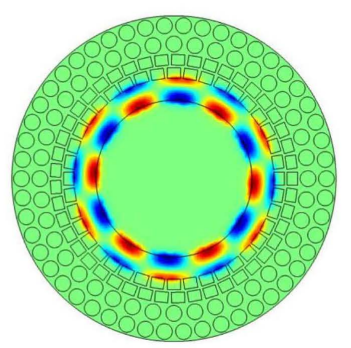

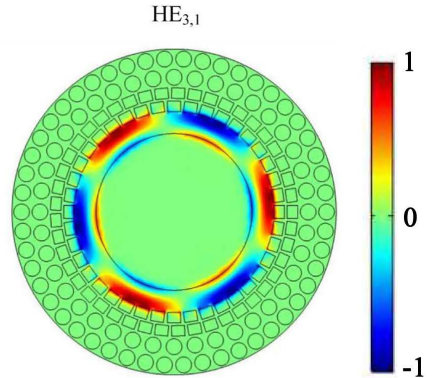

$\mathrm{EH}_{3,1}$

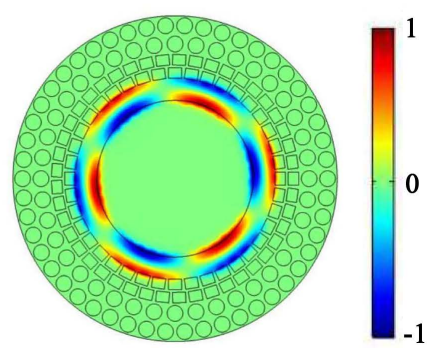

Figure 4. Field intensity distributions in the Ez direction of the partial modes supported by the designed PCF.

fiber are confined to the annular region among the air holes, and both are vortex beams with zero light intensity at the center, but it is difficult to distinguish whether it is EH mode or HE mode. Combined with Figure 4, the EH mode and HE mode of each order can be clearly distinguished. The red and blue area pairs in Figure 4 represent the size of the mode field strength. It can be seen from Figure 4 that there are 12, 6 and 3 groups of red and blue area pairs of $\mathrm{HE}_{12,1}$, $\mathrm{HE}_{6,1}$ and $\mathrm{HE}_{3,1}$ mode. $\mathrm{EH}_{12,1}, \mathrm{EH}_{6,1}$ and $\mathrm{EH}_{3,1}$ mode also have this kind of field distribution. In the field intensity distribution diagram of the Ez direction, the $\mathrm{EH}$ modes are closer to the inner core boundary of the fiber, while the HE modes are closer to the cladding of the fiber [14].

According to Equation (1) and Equation (2), the mode field and phase distribution of the OAM mode generated by the odd mode and even mode superposition of HE mode are as shown in Figure 5. The first and second rows in Figure 5 are mode field and phase distributions of $\mathrm{OAM}_{11}^{+}$mode $\left(\mathrm{OAM}_{11}^{+}=\mathrm{HE}_{21}^{\text {even }}+j \mathrm{HE}_{21}^{\text {odd }}\right)$ and $\mathrm{OAM}_{31}^{+}$mode $\left(\mathrm{OAM}_{31}^{+}=\mathrm{HE}_{41}^{\text {even }}+j \mathrm{HE}_{41}^{\text {odd }}\right)$, respectively. We can see from Figure 5 that the annular distribution of intensity of the OAM mode remains unchanged. The area of the singularity in the center of 

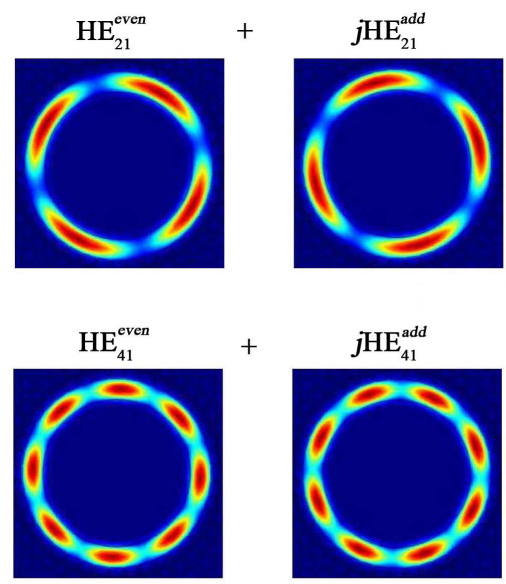

$=$
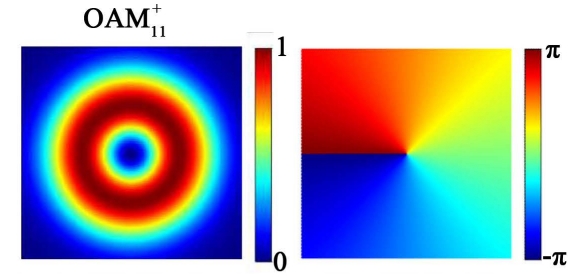

(a)

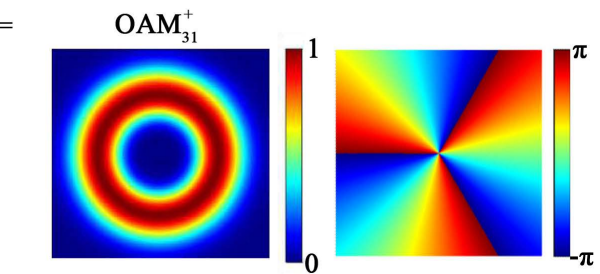

(b)

Figure 5. Changes of the light intensity and phase diagram of the vortex beam superimposed by the vector mode.

the aperture increases as the topological charge increases, and the aperture becomes thinner. Meanwhile, with the increase of topological charge $l$, the phase changes to $2 \pi l$ with a period.

\subsection{The OAM Modes Supported In Designed PCF}

Based on the properties of topological charge and rotation polarization of the OAM mode, the vector modes existing in the PCF are analyzed. It can be known that the topological charge of the vector mode supported by the PCF is $l=1-13$. When the topological charge is $l=1$, the $\mathrm{OAM}_{l, m}$ mode has the same circular polarization and field rotation direction, and 2 OAM modes $\left(\mathrm{OAM}_{ \pm 1,1}\right.$ mode is formed by $\mathrm{HE}_{2,1}$ mode) can be synthesized. When the topological charge number $l \geq 2$, the $\mathrm{OAM}_{l, m}$ mode can be divided into four kinds of information states in which the topological charges are positive and negative and the polarization direction is left and right rotation. Therefore, the PCF structure can contain 50 OAM modes at the wavelength of 1.15 to $2.0 \mu \mathrm{m}(850 \mathrm{~nm}$ ). Compared with the 26 modes in reference [12] and the 46 modes in reference [14], the bands are wider and the number of modes is larger. Table 1 shows OAM modes supported by the PCF and the corresponding vector modes.

\section{The Characteristics of Proposed PCF}

In this part, the characteristics of the fiber were discussed, such as the dispersion, confinement loss, effective mode area and nonlinear coefficient.

\subsection{The Mode Effective Refractive Index}

Figure 6 shows the effective refractive index of the vector mode varies with wavelength. We can know that as the wavelength increases, the mode effective refractive index $n_{\text {eff }}$ decreases in the PCF. In addition, with the increase of the order of the OAM modes, the effective refractive index of the mode decreases 


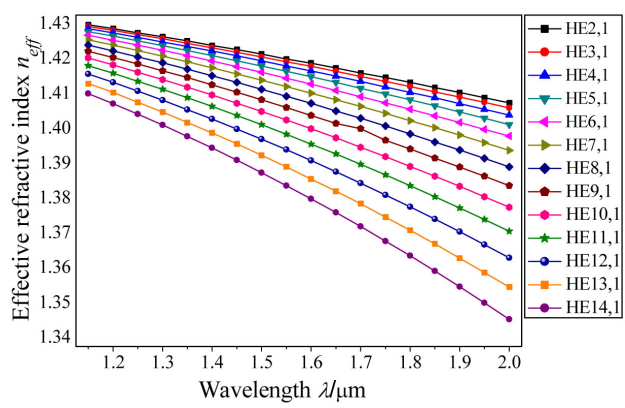

(a)

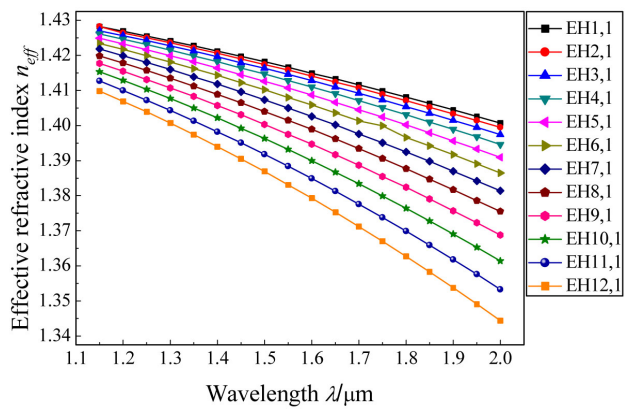

(b)

Figure 6. Variation of the effective refractive index of different vector modes at different wavelengths. (a) variation of HE modes; (b) variation of EH modes.

Table 1. OAM modes are contained in the photonic crystal fiber and the corresponding vector modes.

\begin{tabular}{ccc}
\hline OAM modes & Topological charge $\mid 1$ & Vector modes \\
\hline $\mathrm{OAM}_{ \pm 1,1}^{ \pm}$ & 1 & $\mathrm{HE}_{2,1}$ \\
$\mathrm{OAM}_{ \pm 2,1}^{ \pm}$ & 2 & $\mathrm{HE}_{3,1} \mathrm{EH}_{1,1}$ \\
$\mathrm{OAM}_{ \pm 3,1}^{ \pm}$ & 3 & $\mathrm{HE}_{4,1} \mathrm{EH}_{2,1}$ \\
$\mathrm{OAM}_{ \pm 4,1}^{ \pm}$ & 4 & $\mathrm{HE}_{5,1} \mathrm{EH}_{3,1}$ \\
$\mathrm{OAM}_{ \pm 5,1}^{ \pm}$ & 5 & $\mathrm{HE}_{6,1} \mathrm{EH}_{4,1}$ \\
$\mathrm{OAM}_{ \pm 6,1}^{ \pm}$ & 6 & $\mathrm{HE}_{7,1} \mathrm{EH}_{5,1}$ \\
$\mathrm{OAM}_{ \pm 7,1}^{ \pm}$ & 7 & $\mathrm{HE}_{8,1} \mathrm{EH}_{6,1}$ \\
$\mathrm{OAM}_{ \pm 8,1}^{ \pm}$ & 8 & $\mathrm{HE}_{9,1} \mathrm{EH}_{7,1}$ \\
$\mathrm{OAM}_{ \pm 9,1}^{ \pm}$ & 9 & $\mathrm{HE}_{10,1} \mathrm{EH}_{8,1}$ \\
$\mathrm{OAM}_{ \pm 10,1}^{ \pm}$ & 10 & $\mathrm{HE}_{11,1} \mathrm{EH}_{9,1}$ \\
$\mathrm{OAM}_{ \pm 11,1}^{ \pm}$ & 11 & $\mathrm{HE}_{12,1} \mathrm{EH}_{10,1}$ \\
$\mathrm{OAM}_{ \pm 12,1}^{ \pm}$ & 12 & $\mathrm{HE}_{13,1} \mathrm{EH}_{11,1}$ \\
$\mathrm{OAM}_{ \pm 13,1}^{ \pm}$ & 13 & $\mathrm{HE}_{14,1} \mathrm{EH}_{12,1}$
\end{tabular}

faster and the curve is more inclined. Figure 7 shows the effective refractive index differences $\Delta n_{\text {eff }}$ between the vector modes varies with wavelength. When the wavelength increases, the effective refractive index differences $\Delta n_{\text {eff }}$ become increasingly lager. As can be seen from the standard line with a refractive index 


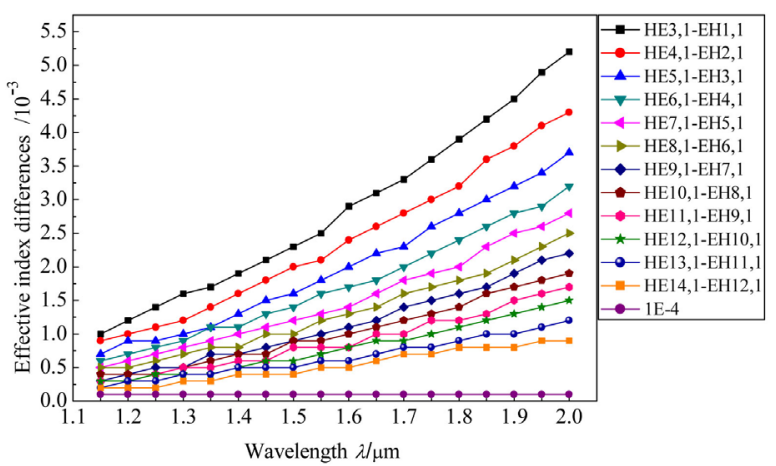

Figure 7. Variation of effective refractive index differences between vector modes at different wavelengths.

difference of $1 \times 10^{-4}$ in Figure 7, the differences $\Delta n_{\text {eff }}$ between the vector modes are greater than $1 \times 10^{-4}$. In particular, the effective refractive index differences $\Delta n_{\text {eff }}$ of $\mathrm{HE}_{3,1}$ and $\mathrm{EH}_{1,1}$ modes can reach $2.6 \times 10^{-3}$ at the wavelength of $1.55 \mu \mathrm{m}$. The larger effective refractive index differences can effectively reduce the modal coupling and form a stable OAM mode transmission without serious crosstalk and mutual coupling.

\subsection{Dispersion Properties}

The fiber dispersion is one of the important parameters in optical waveguides, which has a great impact on the performance of fiber, and it will cause the broadening of optical pulses and further increase the communication rate. PCF can adjust the various structural parameters to make the dispersion value of the photonic crystal fiber maintain a flat characteristic in a relatively wide band. The waveguide dispersion can be expressed as [19]:

$$
D=-\frac{\lambda}{c} \frac{\partial^{2}\left|\operatorname{Re}\left(n_{\text {eff }}\right)\right|}{\partial \lambda^{2}}
$$

where $\operatorname{Re}\left(n_{e f f}\right)$ is the real part of mode effective refractive index.

Figure 8 shows the dispersion versus wavelength curve for each vector mode of the PCF at the wavelength of 1.15 to $2.0 \mu \mathrm{m}(850 \mathrm{~nm})$. It can be seen from Figure 8 that when the incident light wavelength is in the range of 1.15 to 2.0 $\mu \mathrm{m}(850 \mathrm{~nm})$, the dispersion of the low-order mode $(l<6)$ have little change with increasing of wavelength, and the dispersion distribution curve tends to be flat. This phenomenon is beneficial to the stable transmission of the OAM modes in the PCF. The dispersion of the higher-order mode is larger than that of the lower-order mode, and becomes larger as the wavelength increases. This also verifies the unstable transmission of the high-order mode in the fiber. In addition, the dispersion of $\mathrm{HE}_{3,1}$ mode is $46.9649 \mathrm{ps} \cdot(\mathrm{nm} \cdot \mathrm{km})^{-1}$, and the dispersion of $\mathrm{HE}_{4,1}$ mode is $57.4461 \mathrm{ps} \cdot(\mathrm{nm} \cdot \mathrm{km})^{-1}$ at the wavelength of $1.55 \mu \mathrm{m}$.

\subsection{Confinement Loss}

When the light transmits through the core, it will leak into the air holes of the 


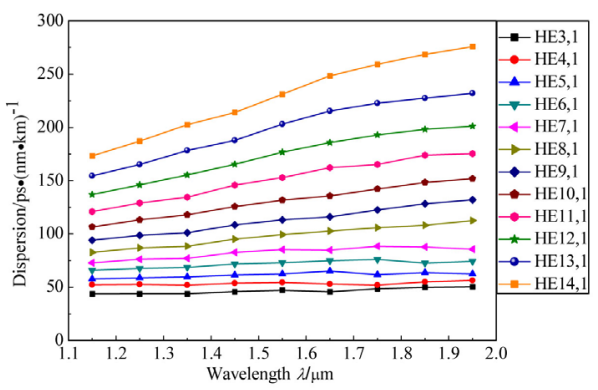

(a)

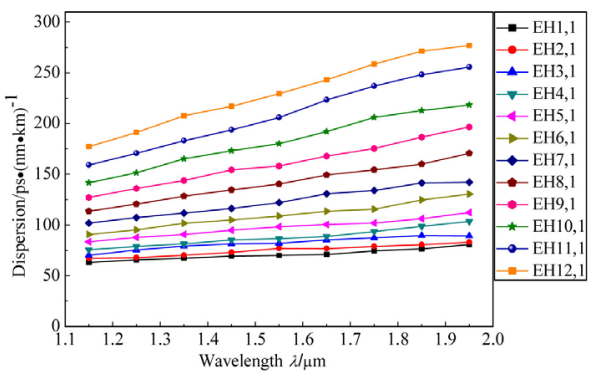

(b)

Figure 8. Dispersion varies with wavelength for the different vector modes. (a) HE modes; (b) EH modes.

cladding in the fiber, resulting in confinement losses in the PCF. The confinement loss $L$ can be expressed as [24]:

$$
L=\frac{2 \pi}{\lambda} \frac{20}{\ln 10} \operatorname{Im}\left(n_{\text {eff }}\right)
$$

where $\operatorname{Im}\left(n_{\text {eff }}\right)$ is the imaginary part of mode effective refractive index, and the unit of confinement loss $L$ is $\mathrm{dB} \cdot \mathrm{m}^{-1}$.

The confinement loss $L$ of the modes at the wavelength of $1.55 \mu \mathrm{m}$ is shown in Table 2. We can know that each vector modes of the PCF has a relatively low confinement loss at a wavelength of $1.55 \mu \mathrm{m}$, and confinement loss is in the range of $10^{-11} \mathrm{~dB} \cdot \mathrm{m}^{-1}$ to $10^{-9} \mathrm{~dB} \cdot \mathrm{m}^{-1}$. This is mainly because each order mode can be well constrained in the fiber core, so the overall confinement loss is relatively ideal. In addition, the confinement loss of $\mathrm{HE}_{4,1}$ mode is only $1.30 \times 10^{-10} \mathrm{~dB} \cdot \mathrm{m}^{-1}$, whose result is four orders of magnitude lower than that in reference [25]. Therefore, it can be seen that the structure of the PCF designed in this study has a small confinement loss when transmitting the vortex beam, which lays a foundation for the stable transmission of the vortex beam in the fiber in the experiment.

\subsection{Effective Mode Area and Nonlinear Coefficient}

The nonlinear coefficient and the effective mode area in the fiber are also important factors influencing the mode transmission. The smaller the nonlinear coefficient is, the better the transmission performance of the fiber communication system. The nonlinear coefficient and the effective mode area are respectively expressed as [26]. 
Table 2. Confinement loss for different vector modes at $1.55 \mu \mathrm{m}$ wavelength in PCF.

\begin{tabular}{|c|c|c|c|c|c|c|}
\hline Vector modes & $\mathrm{HE}_{1,1}$ & $\mathrm{HE}_{2,1}$ & $\mathrm{HE}_{3,1}$ & $\mathrm{HE}_{4,1}$ & $\mathrm{HE}_{5,1}$ & $\mathrm{HE}_{6,1}$ \\
\hline Confinement loss $\left(\mathrm{dB} \cdot \mathrm{m}^{-1}\right)$ & $4.84 \mathrm{E}-10$ & $1.48 \mathrm{E}-10$ & $1.25 \mathrm{E}-09$ & $1.30 \mathrm{E}-10$ & $2.54 \mathrm{E}-10$ & $7.54 \mathrm{E}-11$ \\
\hline Vector modes & $\mathrm{HE}_{7,1}$ & $\mathrm{HE}_{8,1}$ & $\mathrm{HE}_{9,1}$ & $\mathrm{HE}_{10,1}$ & $\mathrm{HE}_{11,1}$ & $\mathrm{HE}_{12,1}$ \\
\hline Confinement loss $\left(\mathrm{dB} \cdot \mathrm{m}^{-1}\right)$ & $2.85 \mathrm{E}-11$ & $3.31 \mathrm{E}-10$ & $5.90 \mathrm{E}-10$ & $7.50 \mathrm{E}-10$ & $3.19 \mathrm{E}-10$ & $3.14 \mathrm{E}-10$ \\
\hline Vector modes & $\mathrm{HE}_{13,1}$ & $\mathrm{HE}_{14,1}$ & $\mathrm{EH}_{1,1}$ & $\mathrm{EH}_{2,1}$ & $\mathrm{EH}_{3,1}$ & $\mathrm{EH}_{4,1}$ \\
\hline Confinement loss $\left(\mathrm{dB} \cdot \mathrm{m}^{-1}\right)$ & $9.08 \mathrm{E}-10$ & $2.04 \mathrm{E}-09$ & $3.70 \mathrm{E}-10$ & $2.85 \mathrm{E}-11$ & $2.85 \mathrm{E}-11$ & $5.90 \mathrm{E}-10$ \\
\hline Vector modes & $\mathrm{EH}_{5,1}$ & $\mathrm{EH}_{6,1}$ & $\mathrm{EH}_{7,1}$ & $\mathrm{EH}_{8,1}$ & $\mathrm{EH}_{9,1}$ & $\mathrm{EH}_{10,1}$ \\
\hline Confinement loss $\left(\mathrm{dB} \cdot \mathrm{m}^{-1}\right)$ & $7.50 \mathrm{E}-10$ & $3.19 \mathrm{E}-10$ & $3.14 \mathrm{E}-10$ & $2.85 \mathrm{E}-11$ & $3.31 \mathrm{E}-10$ & $5.90 \mathrm{E}-10$ \\
\hline Vector modes & $\mathrm{EH}_{11,1}$ & $\mathrm{EH}_{12,1}$ & & & & \\
\hline Confinement loss $\left(\mathrm{dB} \cdot \mathrm{m}^{-1}\right)$ & $7.50 \mathrm{E}-10$ & $3.19 \mathrm{E}-10$ & & & & \\
\hline
\end{tabular}

$$
\begin{gathered}
\gamma=\frac{2 \pi n_{2}}{\lambda \mathrm{A}_{\text {eff }}} \\
\mathrm{A}_{\text {eff }}=\frac{\left(\iint|\mathbf{E}(x, y)|^{2} \mathrm{~d} x \mathrm{~d} y\right)^{2}}{\iint|\mathbf{E}(x, y)|^{4} \mathrm{~d} x \mathrm{~d} y}
\end{gathered}
$$

where $\mathrm{E}(x, y)$ is the distribution of electric field. Since the cladding material is silicon, $n_{2}=2.3 \times 10^{-20} \mathrm{~m}^{2} \cdot \mathrm{w}^{-1}$ is the nonlinear refractive of the fused silica.

The effective mode area and nonlinear coefficient of vector mode are shown in Figure 9 and Figure 10, respectively. It can be known from Figure 9 that as the wavelength and mode order increase, the mode effective area increases because the field strength of the higher-order mode is more likely to leak into the air cladding. On the contrary, the nonlinear coefficient in Figure 10 has the opposite change. The nonlinear coefficients of all vector modes in the PCF are small, ranging from $0.6 \mathrm{~W}^{-1} \cdot \mathrm{km}^{-1}$ to $1.5 \mathrm{~W}^{-1} \cdot \mathrm{km}^{-1}$. This phenomenon is beneficial to the OAM mode transmission in the fiber. The nonlinear coefficient of $\mathrm{HE}_{8,1}$ mode at wavelength $1.55 \mu \mathrm{m}$ is $0.80175 \mathrm{~W}^{-1} \cdot \mathrm{km}^{-1}$. Compared with in references [12] and [27], the nonlinear coefficient is smaller and the nonlinear effect is weaker in this study. Therefore, the better the transmission performance of the fiber communication system in this PCF will be.

\section{Conclusions}

In this study, a PCF structure capable of supporting 50 OAM modes is designed. The fiber characteristics of different modes in the PCF are discussed. Based on the numerical simulation, we can draw the following conclusions:

1) The PCF can support 50 OAM modes in the wavelength range of 1.15 to 2.0 $\mu \mathrm{m}(850 \mathrm{~nm})$, the effective refractive index differences $\Delta n_{\text {eff }}$ of $\mathrm{HE}_{3,1}$ and $\mathrm{EH}_{1,1}$ modes can reach $2.6 \times 10^{-3}$ at the wavelength of $1.55 \mu \mathrm{m}$. The larger effective refractive index differences can effectively separate the vector mode and improve the transmission performance of the OAM mode in fiber. 


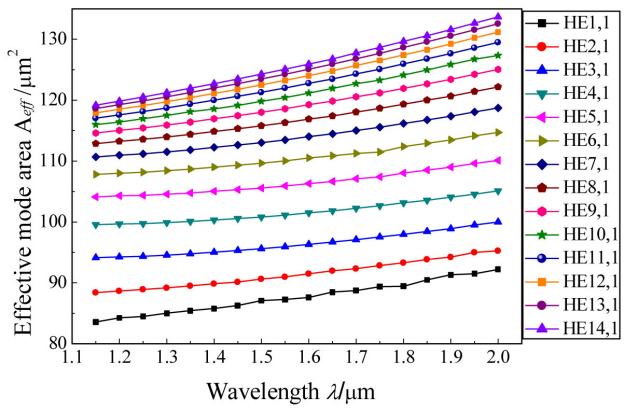

(a)

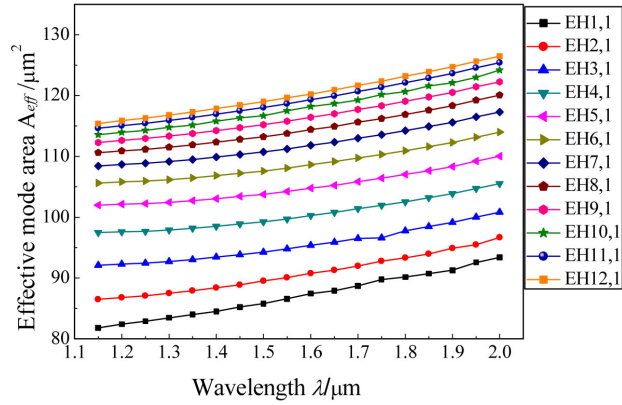

(b)

Figure 9. Variation of effective mode area of different modes at different wavelengths. (a) HE modes; (b) EH modes.

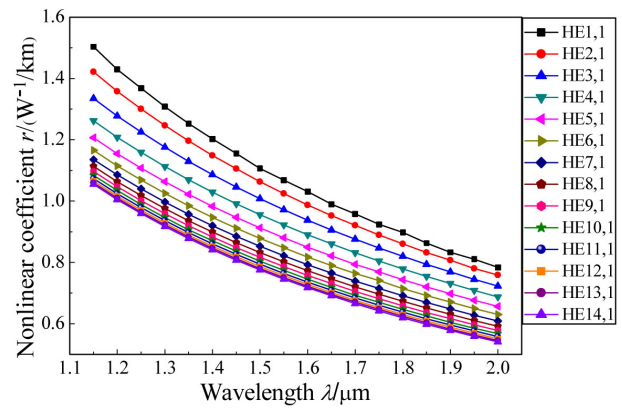

(a)

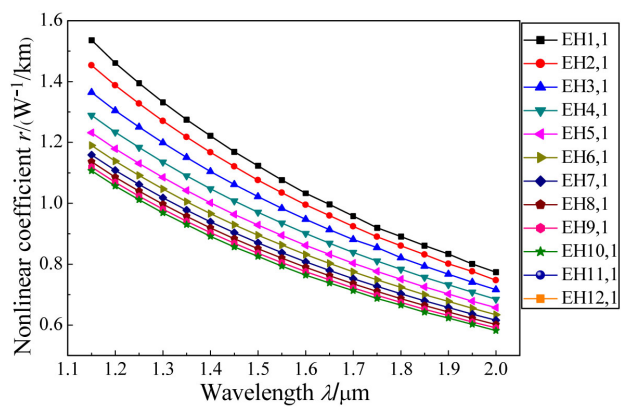

(b)

Figure 10. Variation of nonlinear coefficient of different modes at different wavelengths. (a) Variation of HE modes; (b) variation of EH modes.

2) The dispersion distribution of the low-order mode tends to be flat at the wavelength of $1.15 \mu \mathrm{m}$ to $2.0 \mu \mathrm{m}(850 \mathrm{~nm})$. The confinement losses of all the 
vector mode supported by the fiber are less than $10^{-9} \mathrm{~dB} \cdot \mathrm{m}^{-1}$, and the nonlinear coefficients of all vector modes are between $0.6-1.5 \mathrm{~W}^{-1} \cdot \mathrm{km}^{-1}$. It is beneficial to the stable transmission of the OAM mode. Therefore, by optimizing the fiber parameters of the photonic crystal fiber, the number of OAM modes that supporting transmission can be effectively improved, and various fiber characteristics can be better improved. It is very valuable in large-capacity OAM communication systems that require mode division multiplexing.

\section{Acknowledgements}

This work was supported by Key industry innovation chain project of Shaanxi Province (2017ZDCXL-GY-06-01), Scientific Research Program of Education Department of Shaanxi Province (18JK0341), and Xi'an Science and Technology Innovation Guidance Project (201805030YD8CG14(12)).

\section{Conflicts of Interest}

The authors declare no conflicts of interest regarding the publication of this paper.

\section{References}

[1] Lai, J.S., Wu, B. and Zhao, W.Y. (2014) Application and Analysis of Orbital Angular Momentum Technology in Optical Communication. Telecommunications Science, 30, 46-50.

[2] Allen, L., Beijersbergen, M.W. and Spreeuw, R.J.C. (1992) Orbital Angular Momentum of Light and Transformation of Laguerre-Gaussian Laser Modes. Physical Review A, 45, 8185-8189. https://doi.org/10.1103/PhysRevA.45.8185

[3] Jia, D., Zhang, H. and Zhe, J. (2012) Optical Fiber Amplifiers for Space-Division Multiplexing. Frontiers of Optoelectronics, 5, 351-357. https://doi.org/10.1007/s12200-012-0294-6

[4] Qiao, W., Gao, S. and Lei, T. (2017) Transmission of Orbital Angular Momentum Modes in Grapefruit-Type Microstructure Fiber. Chinese Journal of Lasers, 44, 190-195. https://doi.org/10.3788/CJL201744.0406002

[5] Gregg, P., Kristensen, P. and Golowich, S.E. (2013) Stable Transmission of 12 OAM States in Air-Core Fiber. Cleo. IEEE, Maryland USA, 1-2. https://doi.org/10.1364/CLEO_SI.2013.CTu2K.2

[6] Brunet, C., Vaity, P. and Messaddeq, Y. (2014) Design, Fabrication and Validation of an OAM Fiber Supporting 36 States. Optics Express, 22, 26117-26127. https://doi.org/10.1364/OE.22.026117

[7] Ung, B., Vaity, P. and Wang, L. (2014) Few-Mode Fiber with Inverse-Parabolic Graded-Index Profile for Transmission of OAM-Carrying Modes. Optics Express, 22, 18044-18055. https://doi.org/10.1364/OE.22.018044

[8] Li, S. and Wang, J. (2014) A Compact Trench-Assisted Multi-Orbital-AngularMomentum Multi-Ring Fiber for Ultrahigh-Density Space-Division Multiplexing (19 Rings $\times 22$ OAM Modes). Scientific Reports, 4, 3853-3853. https://doi.org/10.1038/srep03853

[9] Birks, T.A., Knight, J.C. and Russell, P.St.J. (1997) Endlessly Single-Mode Photonic Crystal Fiber. Optics Letters, 22, 961-963. https://doi.org/10.1364/OL.22.000961 
[10] Yue, Y., Zhang, Y. and Yan, Y. (2012) Octave-Spanning Supercontinuum Generation of Vortices in a $\mathrm{As}_{2} \mathrm{~S}_{3}$ Ring Photonic Crystal Fiber. Optics Letters, 37, 1889-1891. https://doi.org/10.1364/OL.37.001889

[11] Wong, G.K.L., Kang, M.S. and Lee, H.W. (2012) Excitation of Orbital Angular Momentum Resonances in Helically Twisted Photonic Crystal Fiber. Science, 337, 446-449. https://doi.org/10.1126/science.1223824

[12] Tian, W., Zhang, H. and Zhang, X. (2016) A Circular Photonic Crystal Fiber Supporting 26 OAM Modes. Optical Fiber Technology, 30, 184-189. https://doi.org/10.1016/j.yofte.2016.07.009

[13] Nandam, A. and Woojin, S. (2018) Spiral Photonic Crystal Fiber Structure for Supporting Orbital Angular Momentum Modes. Optik International Journal for Light and Electron Optics, 169, 361-367. https://doi.org/10.1016/j.ijleo.2018.05.055

[14] Bai, X., Chen, H. and Yang, H. (2018) Design of a Circular Photonic Crystal Fiber with Square Air-Holes for Orbital Angular Momentum Modes Transmission. Optik International Journal for Light and Electron Optics, 158, 1266-1274. https://doi.org/10.1016/j.ijleo.2018.01.015

[15] Ke, X. and Ge, T. (2017) Experiment on Generation of Vortex Light with Few-Mode Fiber. Chinese Journal of Lasers. 44, 182-189. https://doi.org/10.3788/CJL201744.1106004

[16] Ke, X. and Wang, J. (2018) Generation, Transmission, Detection and Application of the Vortex Beam. Science Press, Beijing.

[17] Ramachandran, S., Kristensen, P. and Yan, M. (2009) Generation and Propagation of Radially Polarized Beams in Optical Fibers. Optics Express, 34, 2525-2527. https://doi.org/10.1364/OL.34.002525

[18] Ramachandran, S. and Kristensen, P. (2013) Optical Vortices in Fiber. Nanophotonics, 2, 455-474. https://doi.org/10.1515/nanoph-2013-0047

[19] Dashti, P.Z., Alhassen, F. and Lee, H. (2006) Observation of Orbital Angular Momentum Transfer between Acoustic and Optical Vortices in Optical Fiber. Physical Review Letters, 96, Article ID: 043604. https://doi.org/10.1103/PhysRevLett.96.043604

[20] Inci, H.D. and Ozsoy, S. (2014) Birefringence, Dispersion and Loss Properties for PCFs with Rectangular Air-Hole. Infrared Physics \& Technology, 67, 354-358. https://doi.org/10.1016/j.infrared.2014.08.007

[21] Zhang, X., Yuan, M. and Chang, M. (2018) Characteristics in Square Air Hole Structure Photonic Crystal Fiber. Opto-Electronic Engineering, 45, 23-31.

[22] Kumar, V.V.R.K., George, A.K. and Knight, J.C. (2003) Tellurite Photonic Crystal Fiber. Optics Express, 11, 2641-2645. https://doi.org/10.1364/OE.11.002641

[23] Yajima, T., Yamamoto, J. and Ishii, F. (2013) Low-Loss Photonic Crystal Fiber Fabricated by a Slurry Casting Method. Optics Express, 21, 30500-30506. https://doi.org/10.1364/OE.21.030500

[24] Maji, P. and Chaudhuri, P. (2013) Circular Photonic Crystal Fibers: Numerical Analysis of Chromatic Dispersion and Losses. ISRN Optics, 2013, Article ID: 986924. https://doi.org/10.1155/2013/986924

[25] Zhang, H., Zhang, W. and Xi, L. (2016) A New Type Circular Photonic Crystal Fiber for Orbital Angular Momentum Mode Transmission. IEEE Photonics Technology Letters, 28, 1426-1429. https://doi.org/10.1109/LPT.2016.2551325

[26] Xu, H., Wu, J. and Xu, K. (2011) Ultra-Flattened Chromatic Dispersion Control for Circular Photonic Crystal Fibers. Journal of Optics, 13, 994-1001. 
https://doi.org/10.1088/2040-8978/13/5/055405

[27] Bai, X., Chen, H. and Zhang, L. (2019) Circular Photonic Crystal Fiber Supporting Orbital Angular Momentum Modes Transmission. Infrared and Laser Engineering, 48, 224-231. https://doi.org/10.3788/IRLA201948.0222002 